\title{
Widespread Eosinophilic Pustular Folliculitis in a Nonimmunocompromised Patient
}

\author{
Ana Almodovar-Real ${ }^{a} \quad$ Alejandro Molina-Leyva ${ }^{a}$ \\ María Jose Espiñeira-Carmona ${ }^{a}$ Rosa Ríos-Pelegrinab Ramón Naranjo-Sintes ${ }^{a}$ \\ Husein Husein El-Ahmed ${ }^{c}$ \\ Departments of a Dermatology and ${ }^{b}$ Pathology, San Cecilio University Hospital, Granada, and ${ }^{\mathrm{C}}$ Department of \\ Dermatology, Hospital General de Baza, Granada, Spain
}

\section{Key Words}

Eosinophilic pustular folliculitis · Indomethacin · Dermatosis

\begin{abstract}
Objective: We present a case of eosinophilic pustular folliculitis, a rare dermatosis which is often associated with HIV infection or internal malignancies. Clinical Presentation and Intervention: We report the case of a 66-year-old man with a medical history of hypertension. Histopathological examination showed a dense follicular inflammatory infiltrate with abundant eosinophils. The clinical response to indomethacin was excellent with no recurrence during the follow-up. Conclusion: The patient responded well to indomethacin

(c) 2014 S. Karger AG, Basel
\end{abstract} treatment.

\section{Introduction}

Eosinophilic pustular folliculitis (EPF) is a noninfectious condition characterized by an eosinophilic infiltration of the hair follicles. It was first described by Ofuji et al. [1] in 1970. The etiology of EPF is unknown. Different hypotheses have been postulated including drug hyper-

\begin{tabular}{ll}
\hline KARGER & $\begin{array}{l}\text { ( ) 2014 S. Karger AG, Basel } \\
1011-7571 / 14 / 0235-0475 \$ 39.50 / 0\end{array}$ \\
$\begin{array}{l}\text { E-Mail karger@karger.com } \\
\text { www.karger.com/mpp }\end{array}$ & $\begin{array}{l}\text { This is an Open Access article licensed under the terms of the } \\
\text { Creative Commons Attribution-NonCommercial 3.0 Un- } \\
\text { ported license (CC BY-NC) (www.karger.com/OA-license), } \\
\text { applicable to the online version of the article only. Distribu- } \\
\text { tion permitted for non-commercial purposes only. }\end{array}$
\end{tabular}

sensitivity (i.e. to carbamazepine, minocycline or allopurinol), infections (e.g. Demodex folliculorum, dermatophytes, Pseudomonas aeruginosa and larva migrans) and immunological alterations, which produce a secretion of eosinophilic chemotactic and activation factors [2]. The probable mechanism by which eosinophils infiltrate the pilosebaceous units has not yet been established. The $\mathrm{PGD}_{2} / \mathrm{PGJ}_{2}$-peroxisome proliferator-activated receptor gamma pathway may be involved in the pathogenesis. It induces eotaxin production from sebocytes, which may explain the massive eosinophil infiltrates observed around the pilosebaceous units in EPF [3].

\section{Case Report}

In early October 2012, a 66-year-old man presented with a 5 -day-old itchy rash on his lower limbs, which progressively involved most of his body surface. No mucosal involvement or other systemic manifestations were reported. His medical history revealed hypertension controlled with irbesartan. Physical examination showed scaly annular erythematous-violaceous maculopapules involving the palms of his hands and soles of his feet (fig. 1). On pressure areas, the lesions showed a palpable purpuric macular morphology (fig. 2). Histopathology revealed eosinophilic exocytosis and infundibular spongiosis with eosinophilic and neutrophilic micropustules (fig. 3). In the dermis, an inflammatory infil- 
Fig. 1. Scaly annular erythematous-violaceous maculopapules.

Fig. 2. Lesions show a palpable purpuric macular morphology in the lower limbs.
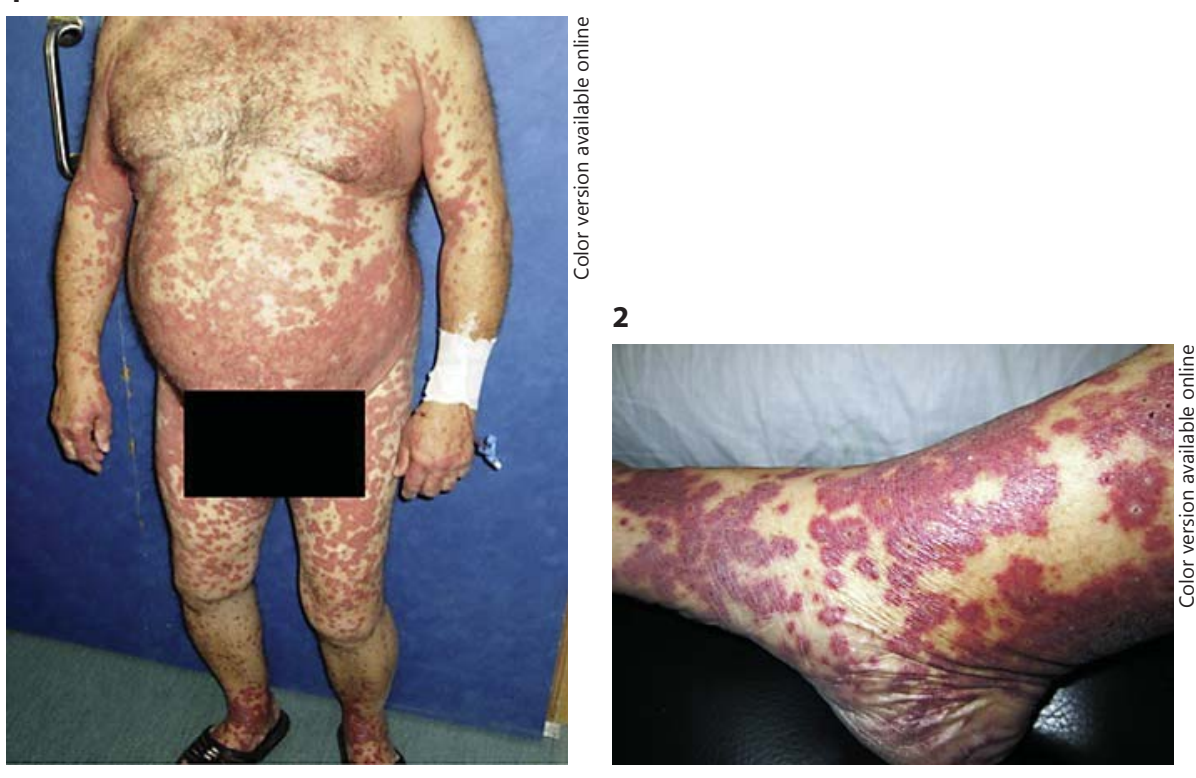



Fig. 3. A histological image shows micropustules with a mixed inflammatory infiltrate consisting predominantly of eosinophils with lymphocytes and neutrophils.

trate of lymphocytes, eosinophils and neutrophils with perivascular and interstitial distribution was observed. In order to exclude a condition of immunosuppression, a complete blood cell count was performed which showed neutrophilia without eosinophilia. Serological tests were negative for human immunodeficiency virus, hepatitis B and C, syphilis, antinuclear antibodies, extractable nuclear antibodies and tumor markers. Immunoelectrophoresis of proteins, a systematic urine test and a peripheral blood smear were normal. Chest computerized tomography and thoracoabdominal magnetic nuclear resonance imaging showed no signs of adenopathies or solid neoplasms. Based on both the positive and the nega- tive findings, a diagnosis of EPF or Ofuji's disease was made. Treatment with indomethacin was initiated and this led to a rapid improvement and the disappearance of the lesions. The patient has remained in good health with no signs of recurrence after 2 years of follow-up.

\section{Discussion}

This is a case of EPF with typical lesions in an otherwise healthy patient, where we were unable to determine a clear etiological factor despite performing several tests and his excellent response to indomethacin. EPF is a rare disease characterized by the presence of itchy papules and pustules in a circinate configuration. Three different subtypes of EPF have been described: classic EPF, immunosuppression-associated EPF (mostly HIV-related or occurring in patients who have received a bone marrow transplant) and childhood EPF. Our case is one of many of EPF in immunocompetent individuals which have been described [4].

In classic EPF, the lesions are characterized by recurrent outbreaks of sterile follicular pustules and papules forming circinate plaques with centrifugal progression which show a predilection for seborrheic areas. Pruritus is present in about $40-50 \%$ of cases, and leukocytosis and eosinophilia have been reported in up to $35 \%$ [2]. In our case, typical circinate lesions were observed, also on the palms of his hands and the soles of his feet, even though these areas have no hair follicles. The lesions displayed neutrophilia without eosinophilia. 
Immunosuppression-associated EPF displays severe itch and discrete erythematous papules with pustules. In childhood, the lesions are on the scalp [5]. In some cases, an association of EPF exacerbation with pregnancy has been found [6].

The skin biopsy of our patient showed eosinophilic exocytosis, infundibular spongiosis and eosinophilic and neutrophilic micropustules. In the dermis, an inflammatory infiltrate of lymphocytes, eosinophils and neutrophils was observed. The epidermis was intact. These histological features characterize EPF [2].

In our patient, we made a differential diagnosis by examining the characteristics of the lesions and the findings of the skin biopsy compared to other conditions. Differential diagnosis should be considered with fungal and viral folliculitis, dermatophyte infections, papular urticaria, graft-versus-host disease (in appropriate settings) and seborrheic dermatitis. Histologically, a differential diagnosis includes various conditions classified under the term 'eosinophilic dermatoses', i.e. arthropod bite reactions, drug-related skin reactions or eosinophilic cellulitis (Wells syndrome) [7].

Different options for EPF management and treatment have been described with variable outcomes [8]. The first choice includes topical corticosteroids. Topical tacrolimus has also been used successfully [9]. Indomethacin is the most effective treatment for typical EPF and it shows clinical improvement in the majority of cases including ours [10]. Our patient remained in good health after achieving resolution with indomethacin. The absence of immunosuppression probably led to a more benign clinical course. Indomethacin is an inhibitor of cyclooxygenases and a potent agonist of the $\mathrm{PGD}_{2}$ receptor CRTH2. It may exert its therapeutic effect via a reduction of CRTH2 expression as well as inhibiting $\mathrm{PGD}_{2}$ synthesis [10]. Other oral treatments with variable outcomes include drugs such as prednisolone, retinoids, colchicine, dapsone, itraconazole, cyclosporine or minocycline [8].

\section{Conclusions}

Our patient with typical EPF had a good clinical response to indomethacin therapy. This report highlights the importance of considering this entity in the workup of pustular and circinate dermatosis.

\section{References}

1 Ofuji S, Ogino A, Horio T, et al: Eosinophilic pustular folliculitis. Acta Derm Venereol 1970;50:195-203.

-2 Nervi SJ, Schwartz RA, Dmochowski M: Eosinophilic pustular folliculitis: a 40-year retrospect. J Am Acad Dermatol 2006;55:285-289.

-3 Nakahigashi K, Doi H, Otsuka A, et al: PGD2 induces eotaxin-3 via PPAR $\gamma$ from sebocytes: a possible pathogenesis of eosinophilic pustular folliculitis. J Allergy Clin Immunol 2012; 129:536-543.

4 Katoh M, Nomura T, Miyachi Y, et al: Eosinophilic pustular folliculitis: a review of the Japanese published works. J Dermatol 2013; 40:15-20.
5 Hernandez-Martin A, Nuno-Gonzalez A, Colmenero I, et al: Eosinophilic pustular folliculitis of infancy: a series of 15 cases and review of the literature. J Am Acad Dermatol 2013;68:150-155.

6 Mabuchi T, Matsuyama T, Ozawa A: Case of eosinophilic pustular folliculitis associated with pregnancy. J Dermatol 2011;38:11911193.

7 Sufyan W, Tan KB, Wong ST, et al: Eosinophilic pustular folliculitis. Arch Pathol Lab Med 2007;131:1598-1601.
8 Fukamachi S, Kabashima K, Sugita K, et al: Therapeutic effectiveness of various treatments for eosinophilic pustular folliculitis. Acta Derm Venereol 2009;89:155-159.

$\checkmark 9$ Hara D, Kuroda K, Mieno H, et al: Treatment of eosinophilic pustular folliculitis with tacrolimus ointment. J Am Acad Dermatol 2004; 51:1040.

10 Satoh T, Shimura C, Miyagishi C, et al: Indomethacin-induced reduction in $\mathrm{CRTH} 2$ in eosinophilic pustular folliculitis (Ofuji's disease): a proposed mechanism of action. Acta Derm Venereol 2010;90:18-22. 